\title{
Clinical and Bacteriological Profile of Neonatal Sepsis in King Khaleed Civilian Hospital, Tabuk, Kingdom of Saudi Arabia
}

\author{
Nagwa Gad Mohamed ${ }^{1}$, Shamina Begum ${ }^{2}$, Mohamed Hamed El-Batanony ${ }^{3}$, \\ Sawsan Mohammed Al Blewi ${ }^{1}$, Walaa Mahmood ${ }^{4}$, Mohammad Zubair ${ }^{2}$ \\ ${ }^{1}$ Department of Paediatrics, Faculty of Medicine, Ministry of Higher Education, University of Tabuk, Tabuk, Kingdom of Saudi Arabia \\ ${ }^{2}$ Department of Medical Microbiology Faculty of Medicine, Ministry of Higher Education, University of Tabuk, Tabuk, Kingdom of Saudi \\ Arabia \\ ${ }^{3}$ King Khaled Hospital, Ministry of Health Tabuk, Tabuk, Kingdom of Saudi Arabia \\ ${ }^{4}$ Department of Family Medicine, Faculty of Medicine, Ministry of Higher Education, University of Tabuk, Tabuk, Kingdom of Saudi Arabia
}

Email address:

mohammad_zubair@yahoo.co.in (M. Zubair)

\section{To cite this article:}

Nagwa Gad Mohamed, Shamina Begum, Mohamed Hamed El-Batanony, Sawsan Mohammed Al Blewi, Walaa Mahmood, Mohammad Zubair. Clinical and Bacteriological Profile of Neonatal Sepsis in King Khaleed Civilian Hospital, Tabuk, Kingdom of Saudi Arabia. European Journal of Preventive Medicine. Vol. 4, No. 1, 2016, pp. 1-6. doi: 10.11648/j.ejpm.20160401.11

\begin{abstract}
Neonatal sepsis is defined as a clinical syndrome of bacteremia with systemic signs and symptoms of infection in the first 28 days of life. Of newborns with early-onset sepsis, $85 \%$ present within 24 hours, 5\% present at $24-48$ hours, and a smaller percentage present within 48-72 hours. The present study included 38 septic neonates. They were divided into two groups: Group with early onset neonatal sepsis (29) and another group with late onset neonatal sepsis (9). The study group with early-onset sepsis showed 18 (62.1\%) males, 11 (37.9\%) females, mean gestational age (weeks) 34.28 \pm 4.7 , mean body weight (gm) 2.1 \pm 0.8 , mean Apgar score at 1 min. 6.7 $\pm 1.8 .21(72.4 \%)$ delivered by CS, $8(27.6 \%)$ delivered by NVD. E. coli was the commonest organism identified in blood culture of septic neonates. Maternal anemia, PROM, and fever were significant risk factors for neonatal sepsis. Prematurity and low birth weights were among the most common neonatal risk factors. Respiratory manifestations were the commonest manifestations of neonatal sepsis in both groups. Treating maternal anemia during pregnancy will help to reduce the incidence of neonatal sepsis $E$. coli is still an important cause of early-onset neonatal sepsis. Blood cultures need to be done strictly before the start of the first dose of antibiotic.
\end{abstract}

Keywords: Neonatal Sepsis, Bacteriological Profile, Tabuk, Kingdom of Saudi Arabia

\section{Introduction}

Neonatal sepsis defined as a clinical syndrome of bacteremia with systemic signs and symptoms of infection in the first 28 days of life. When pathogenic bacteria gain access into the bloodstream, they may cause overwhelming infection without much localization (septicemia) or may be predominantly localized to the lung (pneumonia) or the meninges (meningitis).

Neonatal sepsis (sepsis neonatorum or neonatal septicemia): are terms that are used to describe the systemic response to infection in the newborn infant. The criteria of neonatal sepsis should include the documentation of infection in a newborn infant with a serious systemic illness in which non-specific explanations for the abnormal pathophysiologic state are excluded or unlikely (Gottof, 2000). Neonatal sepsis is a disease of infants who are younger than a month of age, critically ill and have positive blood cultures (Gonzalez et al., 2004). Neonatal sepsis can be classified into two subtypes depending upon whether the onset of symptoms is before 72 hours of life (early-onset neonatal sepsis-EONS) or later (late-onset neonatal sepsis-LONS). These definitions have contributed greatly to diagnosis and treatment by identifying which microorganisms are likely to be responsible for sepsis during these periods and the expected outcomes of infection. Common risk factors associated with the increased severity of the two syndromes are the birth weight and gestational age.

Many focal infections as meningitis, pneumonia and urinary 
tract infection that can occur in other age groups may occur in neonates as well, but infections in neonates have unique elements that differ from those in older age groups. In neonates focal symptoms and signs due to localized infections may be clinically imperceptible and difficult to differentiate on initial presentation from generalized blood stream infections (Baltimore, 2002). Neonatal sepsis is one of the major causes of morbidity and mortality in the newborn. Surviving infants can have significant neurological sequelae as a result of central nervous system (CNS) involvement. Septic shock or hypoxemia can occur secondary to severe parynchymal lung disease (Chako and sohi, 2005). An early, sensitive and specific laboratory test would be helpful to avoid the unnecessary treatment of uninfected patients that may results in over treatment or contribute to antibiotics resistance. Several leukocyte indices and acute-phase protein levels have been evaluated for the diagnosis of sepsis, and measurement of multiple plasma cytokines and leukocyte activation markers have showed promising results (Hodge et al., 2004). However, to date, no single laboratory test has provided rapid and reliable identification of early infected neonates. This inability has led to a search for new diagnostic markers (polin et al., 2003). Because of this, we planned to describe the main clinical and bacteriological profile of neonatal sepsis from King Khaled Civilian Hospital in Tabuk and the associated pathogens.

\section{Methodology}

This study is done on 38 neonates who were admitted to King Khaled Civilian Hospital - Tabuk in the period from June 2014- June 2015 who were diagnosed as neonatal sepsis. Neonates were divided into two groups: Group 1; Early onset group and Group 2; late onset neonatal sepsis group. All groups had been subjected to the following: A: Full detailed history including: maternal risk factors, maternal antibiotics, and cultures are taken on admission before antibiotic taken. B: Through clinical examination \& evaluation for signs of sepsis. Diagnosis of sepsis using sepsis screening: CBC with differential: *Total leucocytic count either leucocytosis > $25,000 / \mathrm{cmm}$ or leucopenia $<5,000 / \mathrm{cmm}$. *Platelets: thrombocytopenia $<100,000 / \mathrm{cmm}$. $*$ I/T ratio: Immature neutrophils (band cells and myelocyte) to total neutrophils ratio $(\mathrm{I} / \mathrm{T})>0.2$ means that immature neutrophils are over 20 percent of the total neutrophils. Acute phase reactants: CRP, Blood culture and sensitivity by standard methods.

Exclusion criteria: The followings are excluded from this study, neonates with:

1. Perinatal asphyxia.

2. Any surgical problems.

Statistical methods.

Descriptive and analytical statistics were performed on an IBM-compatible computer by using SPSS (statistical package for social science). Continous data were presented as mean \pm standard deviation (mean $\pm \mathrm{SD}$ ), categorical data were presented in the form of numbers and percentages. The significant difference between means was determined by student-t-test. Inter-group comparison of categorical data was performed by using chi-square test (X2) value. $\mathrm{P}$ values $<0.05$ were considered significant (Kirkwood \& Sterne, 2003).

\section{Results}

The present study included 38 septic neonates, divided into two groups: Group 1: Group with early onset neonatal sepsis (29) and Group 2: late onset neonatal sepsis (9).

Group 1 showed 18 (62.1\%) males, 11 (37.9\%)females, mean gestational age (weeks) $34.28 \pm 4.7$, mean body weight (gm) $2.1 \pm 0.8$, mean Apgar score at 1 min. 6.7 \pm 1.8 .21 (72.4\%) delivered by CS, $8(27.6 \%)$ delivered by NVD whereas group 2 showed $5(55.6 \%)$ males, $4(44.4 \%)$ females, mean gestational age (wks) 37.7 \pm 2.3 , mean body weight (gm)

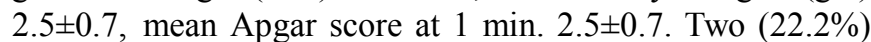
delivered by CS, 7 (77.8\%) delivered by NVD (Table 1).

As regards to maternal risk factors No risk factor in the majority of cases followed by maternal anemia, premature rapture of membrane (PROM) and maternal fever. While (5\%) have antepartum haemorrhage and $(16 \%)$ of the septic group have combined maternal risk factors (table 2, figure 1). As regards to neonatal risk factors we found that prematurity was the highest neonatal risk factor in septic neonates (table 3).

The comparison between the two groups according to the onset of all the clinical manifestation, the number of cases were higher in group 1 compared with group 2 but that difference were statistically insignificant (table $4 \&$ figure 2 ) whereas the comparison between both groups according to sepsis screen revealed that, there is no statistically significant difference regarding all parameters except the hematocrit $(p<0.04)$ which is less in late onset group (Table 5). The most identified organism, especially in the early onset group, is E-coli. The treatment summary of the study was summarized in table 6 .

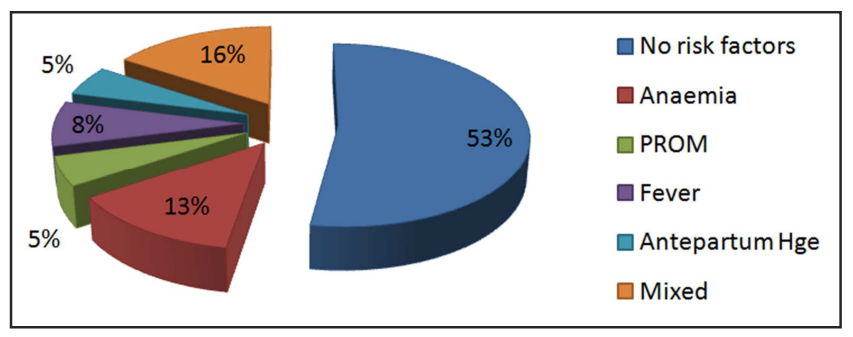

Figure 1. Maternal risk factors in septic neonates.

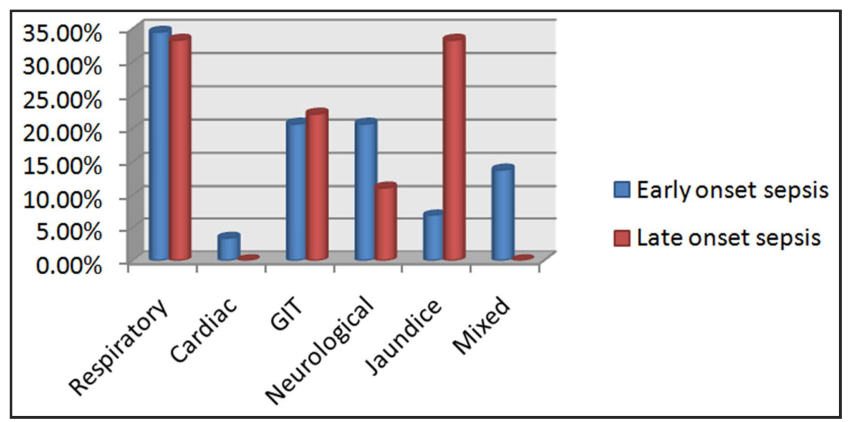

Figure 2. Clinical manifestations of the study groups. 
Table 1. Demographic Data of Both Groups.

\begin{tabular}{|c|c|c|c|c|}
\hline & Early onset sepsis $N=29$ & Late onset sepsis $N=9$ & Ficher exact test & P value \\
\hline \multicolumn{5}{|l|}{ Sex } \\
\hline Male & $18(62.1 \%)$ & $5(55.6 \%)$ & 0.122 & 0.727 \\
\hline Female & $11(37.9 \%)$ & $4(44.4 \%)$ & & \\
\hline \multicolumn{5}{|l|}{ Parity } \\
\hline Primigravida & $2(7.1 \%)$ & $4(44.4 \%)$ & 6.98 & 0.008 \\
\hline Multi & $26(92.9 \%)$ & $5(55.6 \%)$ & & \\
\hline \multicolumn{5}{|l|}{ Mother antibiotic } \\
\hline Yes & $13(44.8 \%)$ & $8(88.9 \%)$ & 5.39 & 0.02 \\
\hline No & $16(55.2 \%)$ & $1(11.1 \%)$ & & \\
\hline \multicolumn{5}{|l|}{ Mode of delivery } \\
\hline NVD & $8(27.6 \%)$ & $7(77.8 \%)$ & 7.24 & 0.007 \\
\hline $\mathrm{CS}$ & $21(72.4 \%)$ & $2(22.2 \%)$ & & \\
\hline \multicolumn{5}{|l|}{ Fate } \\
\hline Died & $6(20.7 \%)$ & $0(0 \%)$ & 2.2 & 0.137 \\
\hline Cured & $23(79.3 \%)$ & $9(100 \%)$ & & \\
\hline Birth weight & $2.1 \pm 0.8$ & $2.5 \pm 0.7$ & 1.178 & 0.247 \\
\hline Mother age at delivery & $30.4 \pm 8.2$ & $26.89 \pm 4.7$ & 1.2 & 0.231 \\
\hline Gestional age & $34.28 \pm 4.7$ & $37.7 \pm 2.3$ & 2.09 & 0.04 \\
\hline APGAR scoring at $1 \mathrm{~min}$ & $6.7 \pm 1.8$ & $2.5 \pm 0.7$ & 2.4 & 0.02 \\
\hline
\end{tabular}

Table 2. Maternal risk factors in septic neonates.

\begin{tabular}{llllll}
\hline & Early onset sepsis $\mathbf{N = 2 9}$ & Late onset sepsis N=9 & Total & Ficher exact test & P value \\
\hline No risk factors & $12(41.4 \%)$ & $8(88.9 \%)$ & $20(52.6 \%)$ & 10.9 & $<0.001$ \\
Anaemia & $5(17.2 \%)$ & 0 & $5(13.2 \%)$ & 1.8 & 0.181 \\
PROM & $2(6.9 \%)$ & 0 & $2(5.3 \%)$ & 0.655 & 0.418 \\
Fever & $2(6.9 \%)$ & $1(11.1 \%)$ & $3(7.9 \%)$ & 0.168 & 0.682 \\
Antepartum Hge & $2(6.9 \%)$ & 0 & $2(5.35)$ & 0.655 & 0.418 \\
Combined & $6(20.7 \%)$ & 0 & $6(15.8 \%)$ & 2.2 & 0.137 \\
\hline
\end{tabular}

Total $\mathrm{x} 2=7.8 \mathrm{p}$ value $=0.17$.

Table 3. Neonatal risk factors in septic neonates.

\begin{tabular}{|c|c|c|c|c|c|}
\hline & Early onset sepsis $\mathbf{N}=29$ & Late onset sepsis $\mathrm{N}=9$ & Total & Ficher exact test & P value \\
\hline Prematurity and low birth weight & $25(86.2 \%)$ & $7(77.8 \%)$ & $32(84.2 \%)$ & $0.367^{*}$ & 0.545 \\
\hline Congenital heart disease & $2(6.9 \%)$ & $1(11.1 \%)$ & $3(7.9 \%)$ & 0.168 & 0.682 \\
\hline Spine and genital anomalies & $2(6.9 \%)$ & 0 & $2(5.3 \%)$ & 0.655 & 0.418 \\
\hline Dysmorphic baby \& ovarian cyst & 0 & $1(11.1 \%)$ & $1(2.6 \%)$ & 0.319 & 0.57 \\
\hline
\end{tabular}

Table 4. Clinical manifestations of the study groups.

\begin{tabular}{|c|c|c|c|c|c|}
\hline Signs & Early onset sepsis $N=29$ & Late onset sepsis $N=9$ & Total & Ficher exact test & $P$ value \\
\hline Respiratory & $10(34.5 \%)$ & $3(33.3 \%)$ & $13(34.2 \%)$ & 0.004 & 0.9 \\
\hline Cardiac & $1(3.4 \%)$ & 0 & $1(2.6 \%)$ & 0.319 & 0.57 \\
\hline GIT & $6(20.7 \%)$ & $2(22.2 \%)$ & $8(21.1 \%)$ & 0.009 & 0.9 \\
\hline Neurological & $6(20.7 \%)$ & $1(11.1 \%)$ & $7(18.4 \%)$ & 0.419 & 0.517 \\
\hline Jaundice & $2(6.9 \%)$ & $3(33.3 \%)$ & $5(13.2 \%)$ & 4.2 & 0.04 \\
\hline Mixed & $4(13.8 \%)$ & 0 & $4(10.5 \%)$ & 1.4 & 0.234 \\
\hline
\end{tabular}

Total $\mathrm{x} 2=5.6 . \mathrm{p}$ value $=0.352$. 
Table 5. Sepsis screen of the study groups.

\begin{tabular}{lllll}
\hline & Early onset sepsis N=29 & Late onset sepsis N=9 & Ficher exact test & P value \\
\hline Hct & $43.9 \pm 12.7$ & $34.01 \pm 6.9$ & $2.23^{*}$ & 0.032 \\
WBCs & $19.02 \pm 16.9$ & $9.1 \pm 1.9$ & $1.7^{* *}$ & $0.923^{* *}$ \\
Neutrophils & $5.98 \pm 3.6$ & $4.7 \pm 3.2$ & $1.39^{* *}$ & 0.363 \\
PLTs & $257.8 \pm 125.4$ & $329.9 \pm 166.3$ & $0.903^{* * *}$ & 0.172 \\
CRP & & & & \\
+ve & $8(27.6 \%)$ & $4(44.4 \%)$ & $3.59^{* * * *}$ \\
-ve & $21(72.4 \%)$ & $5(55.6 \%)$ & & \\
Culture & & & \\
-ve & $24(82.8 \%)$ & $7(77.8 \%)$ & & \\
Ecoli & $4(13.8 \%)$ & $1(11.1 \%)$ & & \\
Staph haemolyticus & 0 & $1(11.1 \%)$ & & \\
Coagulase - ve staph & $1(3.4 \%)$ & 0 & & \\
\hline
\end{tabular}

*T test, $* * \mathrm{U}$ test, $* * *$ Ficher exact test, $* * * * \mathrm{X}^{2}$.

Table 6. Shows the treatment given to the newborns of the two groups.

\begin{tabular}{|c|c|c|c|c|c|}
\hline Antibiotic & Early onset sepsis $\mathbf{N}=29$ & Late onset sepsis $\mathrm{N}=9$ & Total & Ficher exact test & P value \\
\hline Penicillin \& cephalosporin & $15(51.7 \%)$ & $2(22.2 \%)$ & $17(44.7 \%)$ & 2.4 & 0.119 \\
\hline Penicillin \&gentamicin & $12(41.4 \%)$ & $2(22.2 \%)$ & $14(36.8 \%)$ & 1.08 & 0.297 \\
\hline Vancomycin \& cephalosporin & 0 & $3(33.3 \%)$ & $3(7.9 \%)$ & 10.5 & 0.001 \\
\hline Vancomycin \& penicillin & $1(3.4 \%)$ & $1(11.1 \%)$ & $2(5.3 \%)$ & 0.8 & 0.368 \\
\hline Others & $1(3.4 \%)$ & $1(11.1 \%)$ & $2(5.3 \%)$ & 0.8 & 0.368 \\
\hline
\end{tabular}

Total X2=13.2. $\mathrm{p}$ value $=0.01$.

\section{Discussion}

Neonatal sepsis is the major leading cause of neonatal morbidity and mortality worldwide. (Stephen et al., 2008). In developing countries, sepsis is an important contributor to neonatal mortality and morbidity, accounting for just over a third of all neonatal deaths annually, as well as being the major reason for admission to the neonatal unit. (Bang et al., 1999). Early diagnosis and treatment of the newborn infant with suspected sepsis are essential to prevent severe and life threatening complications. It is mandatory to avoid unnecessary use of antibiotics to treat non-infected infants. Thus rapid diagnostic test (s) that differentiate infected from non-infected infants, particularly in the early newborn period, have the potential to make a significant impact on neonatal care (Hodge et al., 2004). Due to the immaturity of their immune system, neonates exhibit a high susceptibility for infections as well as a limited ability to confine these infections with a higher probability of unfavorable outcome. Determining the clinical risk profile sets the stages for the development of strategies directed at preventing poor outcome. (Bang et al., 1999).

This study was designed to study the clinical and bacteriological profile of neonatal sepsis in King Khaled Civilian hospital in Tabuk. This study is done on 38 neonates who were admitted to $\mathrm{KKH}$ with the diagnosis of neonatal sepsis from June 2014 till June 2015. Then newborns were subdivided into 2 groups early onset neonatal sepsis group and late onset neonatal sepsis group. All groups had been subjected to the following: Full detailed history, through clinical examination \& evaluation for signs of sepsis, and laboratory screening of sepsis including CBC, CRP \& blood culture. The results of our study showed that neonatal sepsis was more in the low birth weight babies $(<2.5 \mathrm{~kg})$ than normal birth weight $(2.5$ to $4 \mathrm{~kg}$ ). Moreover, neonatal sepsis was more in those of low gestational age (Premature) than normal gestational age.

The premature newborns were at greater risk for mortality and they had the worst outcome in relation to the full term newborns. This is in agreement of Ahrens et al., 2004 who found that prematurity is the most important neonatal factor predisposing to infection as they have 3-10 fold higher incidence of infection than full-term newborns.

This study revealed that there is an association between sex and neonatal sepsis, sepsis was more in male newborn than females in both early and late onset neonatal sepsis. This was in agreement with Karambin \& Zarkesh (2011) stated that male infants have a fourfold increased risk to develop sepsis than females; this may be due to the presence of gene located on X-chromosome which is involved in the function of the thymus or with synthesis of immunoglobulin. However, Betty and Inderpreet (1997) did not observe any increase in incidence of sepsis among the studied male neonates. This study revealed that there was is an association between mode of delivery and neonatal sepsis. Neonatal sepsis was more in babies of mothers delivered by cesarean section than by normal delivery (in early onset group).

Neonatal sepsis was found in our study to be inversely proportionate to the Apgar scoring at 1minute. Neonatal sepsis was more in babies of multigravida mothers than primigravida. Neonatal sepsis in the early onset group was also found to be more in babies of mothers who did not take antibiotics during delivery.

Regarding maternal risk factors maternal anemia, PROM, 
fever, and anteartumhemorrhage were among the most prominent factors. While $(20.7 \%)$ of the septic group have combined maternal risk factors and $(53 \%)$ with no maternal risk factors. Gluck et al., (2006) found that the incidence of culture-proven sepsis in term neonates with premature rupture of membrane $>24$ hours is $1.3 \%$. This incidence increased to $8.7 \%$ when amnionitis was present. Also, Al-Qa and Al-Awaysheh (2005) noted that the incidence of neonatal sepsis was $11 \%$ among neonates with amaternal history of the premature rapture of membranes who did not receive perinatal antibiotic therapy.

As regards the types of bacteria isolated from blood cultures in the present study of 38 infants who survived beyond 3 days $(21 \%)$ had 1 or more episodes of blood culture-proven late-onset sepsis. coagulase-negative staphylococci accounting for $3.4 \%$ of infections. The rate of infection was inversely related to birth weight and gestational age. Complications of prematurity associated with an increased rate of early-onset sepsis included patent ductus arteriosus, prolonged ventilation, prolonged intravascular access, bronchopulmonary dysplasia, and necrotizing enterocolitis. Infants who developed early-onset sepsis had a significantly prolonged hospital stay (mean length of stay: 10-20 days). They were significantly more likely to die than those who were uninfected $(20.7 \%$ vs $79.3 \%$ ), especially if they were infected with Gram-negative organisms $(82.8 \%)$ or fungi $(32 \%)$.

When we compare our results with that of Barbara J. Stoll. It is somewhat similar but in his results the severity of disease is more in late onset compared to the results that is early onset. Although other studies revealed that the most common etiologies of early onset sepsis include group B streptococci, E. coli, enterococcus species and Listeria monocytogenes (Klein and Marcy, 1999). This study revealed that respiratory manifestations were the commonest manifestations (34.5) with neonatal sepsis followed by neurological manifestations (20.7\%), GIT manifestations (20.7\%). As regard sepsis screen of the study groups: In the early onset sepsis group the Hct is more compared to late onset of sepsis group (the p-value to very less compared to others). The WBC count in early onset is more than late onset and the $\mathrm{T}$ is more than the $\mathrm{p}$-value. Neutrophil are more in early and the p-value is half of the U-test. Platelets is less in early onset than the late and U-test is less than the $\mathrm{p}$-value.

This result agreed with the study of Pherson \& Juul (2005) who found that thrombocytopenia is a manifestation of sepsis and that the severity of thrombocytopenia is generally proportional to the severity of the infectious disease. CRP +ve are less compared to CRP-ve in both early and late onset. The fisher exact test is more compared to P-value. In this study CRP was found to be positive in almost all neonates who were blood culture +ve And this was in agreement of previous studies which found that CRP serves as a good examples of how such biochemical markers aid the neonatal clinicians in distinguishing infected from non infected neonates. (Ehl et al., 2005). Orlikwsky et al, 2004 found that it is not useful as an early phase infection marker but has good diagnostic utilities in the later phase

\section{Summary}

Neonatal sepsis is one of the major causes of morbidity and mortality in the newborn. Every year an estimated 30 million acquire infection \& 1-2millions of these die. Infections are a frequent and important cause of morbidity and mortality in the neonatal period. A variety of factors contributed to this serious disease including maternal risk factors as premature rupture of membrane and others, besides neonatal risk factors as prematurity and invasive procedures. Diagnosis of neonatal sepsis is difficult because the clinical signs are subtle and non-specific and laboratory tests including the "gold standard" blood culture are not always reliable. Many potential markers (acute phase reactants, cell surface markers, and cytokines) are not routinely available to the laboratory, and most likely combinations of markers will ensure a greater diagnostic accuracy. The treatment for suspected neonatal sepsis should be initiated promptly once diagnostic studies have been obtained. This study is designed to study the clinical and bacteriological profile of neonatal sepsis in 38 neonates admitted to KKH in the period between June 2014 to June 2015. This study is done on 38 neonates who were divided into 2 groups: early onset neonatal sepsis and late-onset neonatal sepsis. Each group had been subjected to the following: Full detailed history, through clinical examination $\&$ evaluation for signs of sepsis, and laboratory screening of sepsis including CBC, CRP \& blood culture. The results of our study showed that the rate of infection was inversely related to birth weight and gestational age.

The premature newborns were at greater risk for mortality and they had the worst outcome in relation to the full term newborns. This study revealed that there is an association between sex and neonatal sepsis, sepsis was more in male newborn than females in both early and late onset neonatal sepsis. This study revealed that there was is an association between mode of delivery and neonatal sepsis. Neonatal sepsis was more in babies of mothers delivered by cesarean section than by normal delivery (in early onset group). Regarding maternal risk factors maternal anemia, PROM, fever, and anteartumhemorrhage were among the most prominent factors. Prematurity and low birth weight were among the most common neonatal risk factors. Respiratory manifestations were the commonest manifestations of neonatal sepsis in both groups. E. coli was the commonest organism identified in blood culture of septic neonates. We conclude the following:

- Maternal anemia, PROM and fever were significant risk factors for neonatal sepsis.

- Prematurity and low birth weight was among the most common neonatal risk factors.

- Respiratory manifestations were the commonest manifestations of neonatal sepsis in both groups.

- E. coli was the commonest organism identified in blood culture of septic neonates.

Recommendations: From this study we recommend the following: 
- Treating maternal anemia during pregnancy will help to reduce the incidence of neonatal sepsis

- E. coli is still an important cause of early-onset neonatal sepsis.

- Blood cultures need to be done strictly before the start of the first dose of antibiotic.

- Search for modern molecular biology techniques that offer the prospect of rapid identification of both pathogens and antimicrobial resistance markers.

- Further wide range studies to prove our result of study is recommended.

\section{Acknowledgement}

The authors are thankful to the Deanship of Scientific Research (DSR) University of Tabuk. Tabuk, Saudi Arabia for providing the financial support under research grant no S-1436-0264. We would also like to thank, the consultants for their constant support and clinical evaluation of patients and for dietary advice, monitoring the patients' diet respectively.

\section{References}

[1] Ahrens P, Kattner E, Kohler B. (2004): Mutations of genes involved in the innate immune system as predictors of sepsis in very low birth weight infants. Pediatr Res; 55: 652-656.

[2] Baltimore A. (2002): Perinatal bacterial and fungal infections. In: Hal B. Jenson and Robert S. Baltimore, editors. Pediatric infectious diseases principles and practice. Philadelphia; 96: 1119-33.

[3] Bang AT, Baitule SB, Reddy HM. (1999): Effect of home-based neonatal care and management of sepsis on neonatal mortality: field trial in rural Indian. Lancet; 354: 1955-61.

[4] Betty C and Inderpreet S. (1997): Early onset neonatal sepsis. The Indian Ped; (72): p. 23-26.
[5] Chacko et al (2005): Early onset neonatal sepsis. J Indian pediatr; 17: 538-543.

[6] Ehl S, Gehring B, pohlandtF. (2005): A detailed analysis of changes of serum CRP levels in neonates treated for bacterial infections Eur J pediatr; 158: 238-42.

[7] Hodge D, Puntis JWL. (2004): Diagnosis, preventing, and management of catheter related œ bloodstream infection during long-term parenteral nutrition. Arch Dis Child Fetal Neonatal J; 87: 21 .

[8] Kirkwood BR, Sterne JAC (2003): essential medical statistics, 2nd ed. Oxford: Blackwell scientific.

[9] Klein JO, Marcy SM (1999): Bacterial sepsis and meningitis. Infectious Diseaes of the Fetus and Newborn, Philadelphia WB Saunders. Klein JO, Remington JS. (2001): Current concepts of infections of the Disease of the fetus and Newborn Infant. 5th ed. Philadelphia, WB fetus and newborn infant, Remington JS, Klein JO (editors): Infectious Saunders; 1-23.

[10] Mc Pherson RJ, Juul, Patterns of thrombocytosis and thrombocytopenia in hospitalized neonates. J. perinatol 2005; 25: 166-72.

[11] Mohammad Karambin, MD* and Marjaneh Zarkesh, MD. Entrobacter, the Most Common Pathogen of Neonatal Septicemia in Rasht, Iran. Iran J Pediatr. 2011 Mar; 21 (1): 8387.

[12] Orlikwsky TW, Neunhoeffer F, Goelz R. (2004): Evaluation of IL 8 concentrations in plasma and lysed EDTA-blood in healthy neonates and those with suspected early onset bacterial infection. Pediatr Res; 56: 804-9.

[13] Polin R. (2003): The ins and outs of neonatal sepsis. J Pediatr; 143: 3-4.

[14] Stephen F, Kingsmore, Henry L, Halliday. (2008): Identification of Diagnostic Biomarkers for Infection in Premature Neonates, National Center for Genome Resources, Santa Fe, New Mexico, Molecular \& Cellular Proteomics; 7: 1863-1875. 